\title{
ON THE LITTLEWOOD-PALEY-STEIN $g$-FUNCTION
}

\author{
STEFANO MEDA
}

\begin{abstract}
We consider semigroups $\left(T_{t}\right)$, which are contractive on $L^{p}(M)$ for all $p \in\left[q, q^{\prime}\right]$ and $q \in[1,2)$. We give an example (on symmetric spaces of the noncompact type) which shows that the Littlewood-Paley-Stein $g$-function associated to the infinitesimal generator of $\left(T_{t}\right)$ may be unbounded on $L^{q}(M)$ and on $L^{q^{\prime}}(M)$. We prove that variants of the $g$-function are bounded on these Lebesgue spaces.
\end{abstract}

In this paper we present some new results concerning the $L^{p}$ boundedness of the Littlewood-Paley-Stein $g$-function. Suppose that $\left(T_{t}\right)$ is a $C_{0}$ semigroup of operators on $L^{p}(M)$, where $M$ is a $\sigma$-finite measure space, and let $\alpha \in \mathbf{N}$. The Littlewood-Paley-Stein function $g_{\alpha}$ associated to the given semigroup is defined by the rule

$$
g_{\alpha}(f)=\left(\int_{0}^{\infty}\left|(t A)^{\alpha} T_{t} f\right|^{2} \frac{d t}{t}\right)^{1 / 2}
$$

for all $f$ for which the right-hand side makes sense. In classical cases, where $\left(T_{t}\right)$ is the Poisson semigroup acting on $L^{p}(\mathbf{R})$ or $L^{p}(\mathbf{T})$, the $g$-function is an important tool in Fourier analysis. For instance, it is frequently used to prove multiplier theorems and pointwise convergence results: the reader is referred to [11] for a survey of its role. In a general context, the $g$-function was considered by E. M. Stein; he proved that if $\left(T_{t}\right)$ is a symmetric diffusion semigroup, then $g_{\alpha}$ satisfies the estimate

$$
A_{p}\|f\|_{p} \leq\left\|g_{\alpha}(f)\right\|_{p} \leq B_{p}\|f\|_{p} \quad \forall f \in L^{p}(M),
$$

whenever $p \in(1, \infty)$. A proof of this result can be found in [10]; a simpler and more general approach, via transference techniques, is presented in [2]. A third proof is in [4 and 5]. It is of an entirely different nature, in that it is based on the fact that generators of symmetric diffusion semigroups (and even more general semigroups [3]) have an $H^{\infty}$ functional calculus.

One of the interesting applications of $g$-functions is to functional calculus for infinitesimal generators of $C_{0}$ semigroups. Stein proved a Marcinkiewicz-type result for those as a corollary of the theory he developed for the $g$-function. The relationship between $g$-functions and functional calculus for the infinitesimal generator of $\left(T_{t}\right)$ was investigated in [4 and 5]. Our treatment here owes

Received by the editors March 7, 1994; originally communiated to the Proceedings of the AMS by J. Marshall Ash.

1991 Mathematics Subject Classification. Primary 42B25, 47A60; Secondary 43A85.

Key words and phrases. $g$-function, functional calculus, symmetric spaces.

Research partially supported by the Italian M.U.R.S.T., fondi $40 \%$. 
much to the point of view of [3, 4, and 5]. The motivation for our investigation comes from an interesting example. Suppose that $M$ is a symmetric space of noncompact type, and let $\left(T_{t}\right)$ denote the heat semigroup generated by the Laplace-Beltrami operator on $M$. For any $\theta \in(0,1)$ we consider the modified semigroup $\left(T_{t, \theta}\right)$ defined by the rule $T_{t, \theta}=e^{\theta b t} T_{t}$, where $b$ denotes the bottom of the $L^{2}$-spectrum of the generator. These semigroups have been studied in detail in [1, 6, and 7]. It is well known (see, for instance, [9 or 16] for a proof of this fact) that $T_{t, \theta}$ is contractive on $L^{p}(M)$ if and only if $p \in\left[p_{\theta}, p_{\theta}^{\prime}\right]$, where $p_{\theta}=2 /\left((1-\theta)^{1 / 2}+1\right)$. J. Ph. Anker [1] proved that the standard $g$ function associated to $T_{t, \theta}$ is bounded on $L^{p}(M)$ if $p \in\left(p_{\theta}, p_{\theta}^{\prime}\right)$, by using pointwise estimates for the heat kernel. An interesting question left open is to determine whether the $g$-function is bounded also on $L^{p_{\theta}}(M)$ and $L^{p_{\theta}^{\prime}}(M)$. We remark that the results in the literature do not give any information about this limiting case. Here we give a negative answer to this question. Also, we remark that Anker's result is a simple consequence of the theory developed in [4 and 5], which shows that the result does not depend on the symmetric space structure nor on estimates of the "kernel" of the heat operator.

Our paper is organized as follows. Section 1 contains some results about the $g$-function in an abstract setting. In $\S 2$, we specialize to symmetric spaces of the noncompact type.

We wish to express our gratitude to Michael Cowling for several helpful discussions on the subject of this paper, and for his warm hospitality at the University of New South Wales, where this paper was initiated.

\section{General Results}

Suppose that $A$ is a positive selfadjoint operator on $L^{2}(M)$, where $M$ is a $\sigma$-finite measure space, and let $P_{\lambda}$ be the spectral resolution of the identity for which

$$
A=\int_{0}^{\infty} \lambda d P_{\lambda}
$$

Assume that the family $\left(T_{t}\right)$ of operators defined on $L^{2}(M)$ by the rule

$$
T_{t} f=\int_{0}^{\infty} e^{-t \lambda} d P_{\lambda} f \quad \forall f \in L^{2}(M)
$$

satisfies the estimate

$$
\left\|T_{t} f\right\|_{p} \leq\|f\|_{p} \quad \forall f \in L^{p}(M) \cap L^{2}(M),
$$

whenever $p \in\left[q, q^{\prime}\right]$, where $q \in[1,2)$ and $q^{\prime}$ denotes the index conjugate to $q$. We recall the definition of the Mellin transform on the group $\mathbf{R}^{+}$. If $m \in L^{1}\left(\mathbf{R}^{+}\right)$with respect to the Haar measure $\frac{d \lambda}{\lambda}$, define its Mellin transform $(\mathscr{M} m)$ by the rule

$$
(\mathscr{M} m)(u)=\int_{0}^{\infty} m(\lambda) \lambda^{-i u} \frac{d \lambda}{\lambda} \quad \forall u \in \mathbf{R} .
$$

For every $u \in \mathbf{R}$ define the operator $A^{i u}$ by the rule

$$
A^{i u} f=\int_{0}^{\infty} \lambda^{i u} d P_{\lambda} f \quad \forall f \in L^{2}(M) .
$$


Notice that by spectral theory and the Plancherel formula for the Mellin transform

$$
\begin{aligned}
g_{\alpha}(f) & =\left(\int_{0}^{\infty}\left|(t A)^{\alpha} T_{t} f\right|^{2} \frac{d t}{t}\right)^{1 / 2} \\
& =(2 \pi)^{-1 / 2}\left(\int_{\mathbf{R}}\left|\Gamma(\alpha-i u) A^{i u} f\right|^{2} d u\right)^{1 / 2},
\end{aligned}
$$

for all $f \in L^{2}(M)$. Since $\Gamma$ is a meromorphic function with simple poles at the points $0,-1,-2, \ldots$ and

$$
|\Gamma(\alpha-i u)| \sim e^{-\pi|u| / 2}|u|^{\alpha-1 / 2} \sqrt{2 \pi}
$$

as $|u| \rightarrow \infty$, it is easy to check that the integral above makes sense for all complex $\alpha$ such that $\operatorname{Re}(\alpha)$ is not in $\{0,-1,-2, \ldots\}$. We call this set the "admissible set". Thus, it is reasonable to define $g_{\alpha}$, for all $\alpha$ in the admissible set, by the rule

$$
g_{\alpha}(f)=(2 \pi)^{-1 / 2}\left(\int_{\mathbf{R}}\left|\Gamma(\alpha-i u) A^{i u} f\right|^{2} d u\right)^{1 / 2},
$$

for all $f \in L^{2}(M)$. By spectral theory, it is clear that

$$
\left\|g_{\alpha}(f)\right\|_{2}^{2}=(2 \pi)^{-1} \int_{\mathbf{R}}|\Gamma(\alpha-i u)|^{2}\left\|A^{i u} f\right\|_{2}^{2} d u=C_{\alpha}^{2}\|f\|_{2}^{2},
$$

where $C_{\alpha}^{2}=(2 \pi)^{-1} \int_{\mathbf{R}}|\Gamma(\alpha-i u)|^{2} d u$. It is well known that if $q=1$, i.e., the semigroup is contractive on $L^{p}(M)$ for all $p \in[1, \infty]$, then $g_{\alpha}$ is bounded on $L^{p}(M)$ for all $p \in(1, \infty)$ and $\alpha>0$. For $\alpha$ an integer, this result was proved by Stein [10] for symmetric diffusion semigroups and extended (with a simpler proof) to more general semigroups by R. R. Coifman, R. Rochberg, and G. Weiss [2]. For a positive real $\alpha$ (and still another proof) the result is due to M. G. Cowling [4]. Also, it is not hard to see that in general $g_{\alpha}$ is unbounded on $L^{1}(M)$ and $L^{\infty}(M)$ for all positive real $\alpha$.

We assume that $q \in(1,2)$ and the semigroup is subpositive and contractive on $L^{p}(M)$ for all $p \in\left[q, q^{\prime}\right]$. Under these hypotheses, the imaginary powers of $A$ are bounded on $L^{p}(M)$ for all $p \in\left[q, q^{\prime}\right]$, and the following estimate of their operator norm holds

$$
\left\|A^{i u} \mid\right\|_{p} \leq C_{p}(1+|u|)^{\sigma / 2} \exp (\pi \sigma|u| / 2) \quad \forall u \in \mathbf{R},
$$

where $\sigma=|1 / p-1 / 2| /(1 / q-1 / 2)$. Indeed, if $p=q$, this estimate is a consequence of the transference result [3, Theorem 1]. Since $\left\|A^{i u}\right\| \|_{2}=1$ for all $u \in \mathbf{R}$, the required estimate follows immediately by interpolation.

We present a simple proof of the right-hand inequality of (1) for $p \in\left[2, q^{\prime}\right]$.

Theorem 1.1. Suppose that $p \in\left[2, q^{\prime}\right]$. For every $\alpha$ in the admissible set, define $C_{\alpha, p}$ by the formula

$$
C_{\alpha, p}^{2}=(2 \pi)^{-1} \int_{\mathbf{R}}|\Gamma(\alpha-i u)|^{2}||\left|A^{i u}\right| \|_{p}^{2} d u .
$$

Then we have that

$$
\left\|g_{\alpha}(f)\right\|_{p} \leq C_{\alpha, p}\|f\|_{p} \quad \forall f \in L^{p}(M)
$$


Proof. The case $p=2$ is elementary; thus we may assume $p>2$. Let $r$ be the index conjugate to $p / 2$ and $\phi$ the function in $L^{r}(M)$ such that $\|\phi\|_{r}=1$ and $\left\|g_{\alpha}(f)^{2}\right\|_{p / 2}=\left\langle g_{\alpha}(f)^{2}, \phi\right\rangle$. Then

$$
\begin{aligned}
\left\|g_{\alpha}(f)\right\|_{p}^{2} & =\left\|g_{\alpha}(f)^{2}\right\|_{p / 2}=\left\langle g_{\alpha}(f)^{2}, \phi\right\rangle \\
& =(2 \pi)^{-1} \int_{\mathbf{R}}|\Gamma(\alpha-i u)|^{2}\left\langle\left|A^{i u} f\right|^{2}, \phi\right\rangle d u \\
& \leq(2 \pi)^{-1}\|\phi\|_{r} \int_{\mathbf{R}}|\Gamma(\alpha-i u)|^{2}\left\|A^{i u} f\right\|_{p}^{2} d u \\
& \leq C_{\alpha, p}^{2}\|f\|_{p}^{2},
\end{aligned}
$$

as required.

Corollary 1.2. The following hold:

(i) $g_{\alpha}$ is bounded on $L^{q^{\prime}}(M)$ if $\operatorname{Re}(\alpha)<-1 / 2$ and $\alpha$ is admissible;

(ii) if $p \in\left[2, q^{\prime}\right)$, then $g_{\alpha}$ is bounded on $L^{p}(M)$ for all admissible $\alpha$. Proof. By the asymptotics (2) for the $\Gamma$-function

$$
C_{\alpha, p}^{2} \leq C \int_{\mathbf{R}}(1+|u|)^{2 \operatorname{Re}(\alpha)-1+\sigma} \exp (-\pi|u|(1-\sigma)) d u,
$$

where $\sigma=(1 / 2-1 / p) /\left(1 / 2-1 / q^{\prime}\right)$. This integral is finite if $p \in\left[2, q^{\prime}\right)$ or if $p=q^{\prime}$ and $\operatorname{Re}(\alpha)<-1 / 2$, as required.

Unfortunately, we do not have such an elementary proof for the case where $p \in[q, 2)$. However, we have a reasonably simple proof, which is modelled over the proof of [5, Theorem 6.8]. We need first a couple of lemmata. The first is a randomization lemma; for the proof, the reader may consult [10 or 5].

Lemma 1.3. Suppose that $1<p<\infty$ and that $\left(f_{j}\right)_{j \in \mathbf{Z}}$ is a sequence of functions in $L^{p}(M)$. Then

$$
\left\|\left(\sum_{j=-\infty}^{\infty}\left|f_{j}\right|^{2}\right)^{1 / 2}\right\|_{p} \leq C \sup _{\left|a_{j}\right| \leq 1}\left\|\sum_{j=-\infty}^{\infty} a_{j} f_{j}\right\|_{p} .
$$

Let $\gamma_{\pi / 2}$ be the set $\{z \in \mathbf{C}: \operatorname{Re}(z)>0\}$, and denote by $S_{\pi / 2}$ the strip $\{z \in \mathbf{C}:|\operatorname{Im}(z)|<\pi / 2\}$. Let $h$ be a smooth, compactly supported function on the real line; and for every $k$ in $\mathbf{Z}$, define $h_{k}$ by the rule $h_{k}(u)=h(u-k)$ for every $u \in \mathbf{R}$. Choose $h$ such that

$$
\sum_{k=-\infty}^{\infty} h_{k}(u)=1 \quad \forall u \in \mathbf{R} .
$$

For every $j$ and $k$ in $\mathbf{Z}$ define the function $b_{j k}$ by the formula

$$
b_{j k}(\zeta)=\int_{\mathbf{R}} h_{k}(u) \Gamma(\alpha-i u) e^{-i j u} \zeta^{i u} d u \quad \forall \zeta \in \gamma_{\pi / 2} .
$$

Notice that for every $\alpha$ in the admissible set, $b_{j k}$ is holomorphic and bounded in the set $\gamma_{\pi / 2}$. For every $j$ and $k$ in $\mathbf{Z}$, let $B_{j k}$ be the function defined by the rule

for almost every $y$ in $\mathbf{R}$.

$$
B_{j k}(y)=\lim _{x \rightarrow 0^{+}} b_{j k}(x+i y)
$$


Lemma 1.4. Let $\left(a_{j}\right)_{j \in \mathrm{Z}}$ be a sequence of complex numbers such that $\sup _{j}\left|a_{j}\right| \leq$ 1. Then the function $m_{k}: \mathbf{R} \rightarrow \mathbf{C}$ defined by the rule

$$
m_{k}=\sum_{j=-\infty}^{\infty} a_{j} B_{j k}
$$

is an $L^{p}$ multiplier for every $p \in(1, \infty)$ and for every $\varepsilon>0$ satisfies the estimate

$$
\left\|m_{k}\right\|_{\mathscr{A}_{p}(\mathbf{R})} \leq C_{p, \varepsilon} \gamma_{k}(\operatorname{Re}(\alpha)+\varepsilon)
$$

where $\gamma_{k}: \mathbf{R} \rightarrow \mathbf{R}^{+}$is defined by the formula

$$
\gamma_{k}(\omega)=(1+|k|)^{\omega} \log ^{2}(2+|k|) .
$$

Proof. In order to obtain cleaner formulae, the following definition is useful:

$$
\omega_{\alpha}^{-}=\operatorname{Re}(\alpha)-1 / 2 \text { and } \omega_{\alpha}^{+}=\operatorname{Re}(\alpha)+1 / 2 .
$$

Also, let $\varphi(u)=|\Gamma(\alpha-i u)|+\left|\Gamma^{\prime}(\alpha-i u)\right|+\left|\Gamma^{\prime \prime}(\alpha-i u)\right|$. We remark that

$$
\varphi(u) \leq C(1+|u|)^{\omega_{a}^{-}} \log ^{2}(2+|u|) e^{-\pi|u| / 2} \quad \forall u \in \mathbf{R},
$$

by elementary analytic function theory. We shall prove that $m_{k}$ satisfies the hypotheses of the Hörmander multiplier theorem. Indeed, for every $x$ in $\mathbf{R}$ we have that

$$
\begin{aligned}
\left|B_{j k}\left(e^{x}\right)\right| & =\left|b_{j k}\left(e^{x+i \pi / 2}\right)\right|=\left|\int_{\mathbf{R}} h_{k}(u) \Gamma(\alpha-i u) e^{i(x+i \pi / 2-j) u} d u\right| \\
& \leq \int_{-k-2}^{-k+2}|\Gamma(\alpha-i u)| e^{\pi|u| / 2} d u \\
& \leq C \int_{-k-2}^{-k+2}(1+|u|)^{\omega_{\alpha}^{-}} d u \\
& \leq C(1+|k|)^{\omega_{\alpha}^{-}}
\end{aligned}
$$

and

$$
\begin{aligned}
\left|B_{j k}\left(e^{x}\right)\right| & =\left|\frac{1}{(j-x)^{2}} \int_{\mathbf{R}} \frac{d^{2}}{d u^{2}}\left(h_{k}(u) \Gamma(\alpha-i u)\right) e^{i(x+i \pi / 2-j) u} d u\right| \\
& \leq \frac{C}{|j-x|^{2}} \int_{-k-2}^{-k+2} \varphi(u) e^{\pi|u| / 2} d u \\
& \leq \frac{C}{|j-x|^{2}} \int_{-k-2}^{-k+2}(1+|u|)^{\omega_{\alpha}^{-}} \log ^{2}(2+|u|) d u \\
& \leq C \frac{(1+|k|)_{\alpha}^{-} \log ^{2}(2+|k|)}{|j-x|^{2}} .
\end{aligned}
$$

Moreover,

$$
\begin{aligned}
\left|\frac{d}{d x}\left(B_{j k} \circ \exp \right)(x)\right| & =\left|\int_{\mathbf{R}} h_{k}(u) \Gamma(\alpha-i u) i u e^{i(x+i \pi / 2-j) u} d u\right| \\
& \leq \int_{-k-2}^{-k+2}|\Gamma(\alpha-i u)||u| e^{\pi|u| / 2} d u \\
& \leq C \int_{-k-2}^{-k+2}(1+|u|)^{\omega_{a}^{+}} d u \\
& \leq C(1+|k|)^{\omega_{a}^{+}}
\end{aligned}
$$


and

$$
\begin{aligned}
\left|\frac{d}{d x}\left(B_{j k} \circ \exp \right)(x)\right| & =\left|\frac{1}{(j-x)^{2}} \int_{\mathbf{R}} \frac{d^{2}}{d u^{2}}\left(h_{k}(u) \Gamma(\alpha-i u)\right) i u e^{i(x+i \pi / 2-j) u} d u\right| \\
& \leq \frac{C}{|j-x|^{2}} \int_{-k-2}^{-k+2} \varphi(u)|u| e^{\pi|u| / 2} d u \\
& \leq \frac{C}{|j-x|^{2}} \int_{-k-2}^{-k+2}(1+|u|)^{\omega_{\alpha}^{+}} \log ^{2}(2+|u|) d u \\
& \leq C \frac{(1+|k|)_{\alpha}^{+} \log ^{2}(2+|k|)}{|j-x|^{2}}
\end{aligned}
$$

We claim that

$$
\left(\int_{R / 2}^{2 R}\left|m_{k}(y)\right|^{2} \frac{d y}{y}\right)^{1 / 2} \leq C \gamma_{k}\left(\omega_{\alpha}^{-}\right) \quad \forall R \in \mathbf{R}^{+}
$$

and

$$
\left(\int_{R / 2}^{2 R}\left|y m_{k}^{\prime}(y)\right| \frac{d y}{y}\right)^{1 / 2} \leq C \gamma_{k}\left(\omega_{\alpha}^{+}\right) \quad \forall R \in \mathbf{R}^{+} .
$$

Indeed,

$$
\begin{aligned}
& \left(\int_{R / 2}^{2 R}\left|m_{k}(y)\right|^{2} \frac{d y}{y}\right)^{1 / 2}=\left(\int_{R / 2}^{2 R}\left|\left(\sum_{|j-\log y|<1}+\sum_{|j-\log y| \geq 1}\right) a_{j} B_{j k}(y)\right|^{2} \frac{d y}{y}\right)^{1 / 2} \\
& \quad \leq C\left(\int_{R / 2}^{2 R}\left|(1+|k|)^{\omega_{\alpha}^{-}}+\sum_{|j-\log y| \geq 1} \frac{(1+|k|)^{-} \log ^{2}(2+|k|)}{|j-\log y|^{2}}\right|^{2} \frac{d y}{y}\right)^{1 / 2} \\
& \leq C \gamma_{k}\left(\omega_{\alpha}^{-}\right)\left(\int_{R / 2}^{2 R}\left|1+\sum_{|j-\log y| \geq 1} \frac{1}{|j-\log y|^{2}}\right|^{2} \frac{d y}{y}\right)^{1 / 2} \\
& \leq C \gamma_{k}\left(\omega_{\alpha}^{-}\right),
\end{aligned}
$$

and, similarly,

$$
\begin{aligned}
& \left(\int_{R / 2}^{2 R}\left|y m_{k}^{\prime}(y)\right|^{2} \frac{d y}{y}\right)^{1 / 2}=\left(\int_{R / 2}^{2 R}\left|\left(\sum_{|j-\log y|<1}+\sum_{|j-\log y| \geq 1}\right) a_{j} y B_{j k}^{\prime}(y)\right|^{2} \frac{d y}{y}\right)^{1 / 2} \\
& \quad \leq C\left(\int_{R / 2}^{2 R}\left|(1+|k|)^{\omega_{\alpha}^{+}}+\sum_{|j-\log y| \geq 1} \frac{(1+|k|)_{\alpha}^{+} \log ^{2}(2+|k|)}{|j-\log y|^{2}}\right|^{2} \frac{d y}{y}\right)^{1 / 2} \\
& \quad \leq C \gamma_{k}\left(\omega_{\alpha}^{+}\right)\left(\int_{R / 2}^{2 R}\left|1+\sum_{|j-\log y| \geq 1} \frac{1}{|j-\log y|^{2}}\right|^{2} \frac{d y}{y}\right)^{1 / 2} \\
& \leq C \gamma_{k}\left(\omega_{\alpha}^{+}\right),
\end{aligned}
$$


as claimed. If $\eta$ is a smooth compactly supported function on $\mathbf{R}^{+}$, supported in $[1,4]$ and equal to 1 in $[2,3]$, then this calculation implies that

$$
\sup _{R>0}\left\|\eta(|\cdot|) m_{k}(R \cdot)\right\|_{L^{2}(\mathbf{R})} \leq C \gamma_{k}\left(\omega_{\alpha}^{-}\right)
$$

and

$$
\sup _{R>0}\left\|\eta(|\cdot|) m_{k}(R \cdot)\right\|_{H^{1}(\mathbf{R})} \leq C \gamma_{k}\left(\omega_{\alpha}^{+}\right),
$$

where $H^{1}(\mathbf{R})$ denotes the usual Sobolev space on the real line. By interpolation, we obtain that for every $\varepsilon>0$

$$
\sup _{R>0}\left\|\eta(|\cdot|) m_{k}(R \cdot)\right\|_{H^{1 / 2+\varepsilon}(\mathbf{R})} \leq C \gamma_{k}(\operatorname{Re}(\alpha)+\varepsilon) ;
$$

now, by (a refined version of) the Hörmander multiplier theorem, this estimate implies that

as required.

$$
\left\|m_{k}\right\|_{\mathscr{M}_{p}(\mathbf{R})} \leq C_{p, \varepsilon} \gamma_{k}(\operatorname{Re}(\alpha)+\varepsilon)
$$

Theorem 1.5. The following hold:

(i) $g_{\alpha}$ is bounded on $L^{q}(M)$ if $\operatorname{Re}(\alpha)<-1$ and $\alpha$ is admissible;

(ii) if $p \in(q, 2]$, then $g_{\alpha}$ is bounded on $L^{p}(M)$ for all admissible $\alpha$.

Proof. We first prove (i). The idea of the proof is taken from [5, Theorem 6.8]. By applying Minkowski's inequality followed by Plancherel's formula, we obtain that

$$
\begin{aligned}
\left\|g_{\alpha}(f)\right\|_{q} & \leq \sum_{k=-\infty}^{\infty}\left\|\left(\int_{\mathbf{R}}\left|h_{k}(u) \Gamma(\alpha-i u) A^{i u} f\right|^{2} d u\right)^{1 / 2}\right\|_{q} \\
& \leq \sum_{k=-\infty}^{\infty}\left\|\left(\sum_{j=-\infty}^{\infty}\left|b_{j k}(A) f\right|^{2}\right)^{1 / 2}\right\|_{q} .
\end{aligned}
$$

Now, by Lemma 1.3 the last expression is dominated by a constant times

$$
\sum_{k=-\infty}^{\infty} \sup _{\left|a_{j}\right| \leq 1}\left\|\sum_{j=-\infty}^{\infty} a_{j} b_{j k}(A) f\right\|_{q} \text {. }
$$

We apply the transference result of Cowling [3, Theorem 1 and Lemma 1.4] and obtain that the operator $m_{k}(A)$, defined by the formula

$$
m_{k}(A)=\sum_{j=-\infty}^{\infty} a_{k} b_{j k}(A) f
$$

satisfies the estimate

$$
\left\|m_{k}(A)\right\|_{q} \leq C_{q, \varepsilon} \gamma_{k}(\operatorname{Re}(\alpha)+\varepsilon)
$$

so that

$$
\left\|g_{\alpha}(f)\right\|_{q} \leq C_{q, \varepsilon}\|f\|_{q} \sum_{k=-\infty}^{\infty} \gamma_{k}(\operatorname{Re}(\alpha)+\varepsilon)
$$

The last series converges provided that $\operatorname{Re}(\alpha)<-1-\varepsilon$, as required. This concludes the proof of (i). 
We now prove (ii). Observe that, by spectral theory,

$$
\left\||| m_{k}(A)\left|\|_{2}=\sup _{\lambda>0}\right| m_{k}(\lambda) \mid .\right.
$$

By an argument similar to that of the lemma, we deduce that

$$
\left\|\mid m_{k}(A)\right\|_{2} \leq C \log ^{2}(2+|k|)(1+|k|)^{\operatorname{Re}(\alpha)+\varepsilon} \exp (-\pi|k| / 2) \quad \forall k \in \mathbf{Z} ;
$$

therefore, by the Riesz-Thorin interpolation theorem we may conclude that if $p \in(q, 2)$, then

$$
\left\|\left|m_{k}(A) \|\right|_{2} \leq C \log ^{2 \sigma}(2+|k|)(1+|k|)^{\sigma(\operatorname{Re}(\alpha)+\varepsilon)} \exp (-\sigma \pi|k| / 2),\right.
$$

where $\sigma=(1 / q-1 / p) /(1 / q-1 / 2)$. Thus, arguing as in (i), we obtain that

$$
\left\|g_{\alpha}(f)\right\|_{p} \leq C\|f\|_{p} \sum_{k=-\infty}^{\infty}\left(\gamma_{k}(\operatorname{Re}(\alpha)+\varepsilon)\right)^{\sigma} \exp (-\sigma \pi|k| / 2) ;
$$

the last series converges for all admissible $\alpha$, thereby concluding the proof of (ii).

The proof of the theorem is now complete.

\section{SYMMETRIC SPACES}

In this section $M$ will denote a symmetric space of the noncompact type. More explicitly, let $G$ and $K$ be a connected noncompact semisimple Lie group with finite center and a maximal compact subgroup thereof, and consider the symmetric space $G / K$, also denoted by $M$. There is a canonical invariant Riemannian metric on $M$; denote by $-\mathscr{L}_{0}$ the associated Laplace-Beltrami operator. By general nonsense, $\mathscr{L}_{0}$ is positive and essentially selfadjoint on $C_{c}^{\infty}(M)$; let $\mathscr{L}$ be the unique selfadjoint extension of $\mathscr{L}_{0}$ and $\left\{P_{\lambda}\right\}$ the spectral resolution of the identity for which

$$
\mathscr{L} f=\int_{b}^{\infty} \lambda d P_{\lambda} f \quad \forall f \in \operatorname{Dom}(\mathscr{L}),
$$

where the bottom of the spectrum, $b$, is equal to $\langle\rho, \rho\rangle, \rho$ being the usual half-sum of the positive roots. Consider the heat semigroup generated by $\mathscr{L}$, namely

$$
T_{t} f=\int_{b}^{\infty} \exp (-t \lambda) d P_{\lambda} f
$$

For every $\theta \in(0,1)$ we consider the modified semigroup $T_{t, \theta}$ defined by the rule $T_{t, \theta}=e^{\theta b t} T_{t}$. Clearly, $T_{t, \theta}$ is generated by $\mathscr{L}_{\theta}$, where $\mathscr{L}_{\theta}=\mathscr{L}-\theta b$. For any admissible $\alpha$, let $g_{\alpha, \theta}$ be defined by the rule

$$
g_{\alpha, \theta}(f)=(2 \pi)^{-1 / 2}\left(\int_{\mathbf{R}}\left|\Gamma(\alpha-i u) \mathscr{L}_{\theta}^{i u} f\right|^{2} d u\right)^{1 / 2} .
$$

Anker [1] proved that if $\alpha$ is a positive integer, then $g_{\alpha, \theta}$ is bounded on $L^{p}(M)$ if $p \in\left(p_{\theta}, p_{\theta}^{\prime}\right)$. Apparently, his methods do not give any information about the boundedness of $g_{\alpha}$ on $L^{p_{\theta}}(M)$ and $L^{p_{\theta}^{\prime}}(M)$. Our aim is to prove some endpoint results for $g_{\alpha, \theta}$.

We first recall some basic facts about the semigroup $\left(T_{t, \theta}\right)$ and its generator $\mathscr{L}_{\theta}$. It is well known (see, for example, [6] for a proof of this fact) that $T_{t, \theta}$ is 
contractive on $L^{p}(M)$ if and only if $p \in\left[p_{\theta}, p_{\theta}^{\prime}\right]$, where $p_{\theta}=2 /\left((1-\theta)^{1 / 2}+1\right)$. Also, $\mathscr{L}_{\theta}$ possesses a holomorphic functional calculus as illustrated by the following result, which was proved in [6] and is included here for the reader's convenience.

Theorem 2.1. Suppose that $0 \leq \theta<1$ and that $p_{\theta} \leq p \leq p_{\theta}^{\prime}$ and $1<p<\infty$. Suppose also that $m$ is holomorphic and bounded in the right half-plane, and denote by $M$ the nontangential limit of $m$ at the imaginary axis, i.e., $M(y)=$ $\lim _{x \rightarrow 0+} m(x+i y)$ for almost every $y$ in $\mathbf{R}$.

(i) If $M$ is an $L^{p}(\mathbf{R})$-Fourier multiplier of norm $B$, then the operator $m\left(\mathscr{L}_{\theta}\right)$ is bounded on $L^{p}(X)$. Moreover, $\left\|\left|m\left(\mathscr{L}_{\theta}\right)\right|\right\|_{p} \leq B$.

(ii) If $\eta$ is a smooth, compactly supported function on $\mathbf{R}^{+}$, supported in [1, 4] and equal to 1 in [2,3], and if there exists a constant $B$ such that

$$
\|\eta(\cdot) M(R \cdot)\|_{\Lambda_{2,1}^{1 / 2}(\mathbf{R})} \leq B \quad \forall R \in \mathbf{R}^{+}
$$

(where $\Lambda_{2,1}^{1 / 2}(\mathbf{R})$ denotes the usual Besov-Lipschitz space on the real line), then the operator $m\left(\mathscr{L}_{\theta}\right)$ is bounded on $L^{p}(X)$. Moreover, $\left\|m\left(\mathscr{L}_{\theta}\right) \mid\right\|_{p} \leq C B$.

It was proved in [6] that there exist constant $C_{\theta}$ and $C_{\theta}^{\prime}$ such that

$$
C_{\theta} \exp (\pi|u| / 2) \leq||\left|\mathscr{L}_{\theta}^{i u}\right| \|_{p_{\theta}} \leq C_{\theta}^{\prime}(1+|u|)^{1 / 2} \exp (\pi|u| / 2) \quad \forall u \in \mathbf{R} .
$$

Since $\left\|\left|\mathscr{L}_{\theta}^{i u}\right|\right\|_{2}=1$, we get by interpolation and duality that for all $p \in\left(p_{\theta}, p_{\theta}^{\prime}\right)$

$$
\left\|\mathscr{L}_{\theta}^{i u} \mid\right\|_{p} \leq C_{\theta, p}(1+|u|)^{\sigma / 2} \exp (\pi \sigma|u| / 2) \quad \forall u \in \mathbf{R},
$$

where $\sigma=|1 / p-1 / 2| /\left(1 / p_{\theta}-1 / 2\right)$.

In order to make the proof of the main result of this section readable, we have to introduce more notation. However, to avoid wasting space, we shall be very concise. The reader who is familiar with the subject will find our notation standard; the reader who is not an expert is urged to consult the treatise [8] or, for a more concise exposition, $[6, \S 2]$.

Let $G$ be a noncompact semisimple Lie group with finite centre, write $\mathfrak{g}=$ $\mathfrak{k} \oplus \mathfrak{p}$ for a Cartan decomposition of the Lie algebra $\mathfrak{g}$ of $G$, and let $\mathfrak{a}$ be a maximal abelian subspace of $\mathfrak{p}$. Recall that the Killing form $B(\cdot, \cdot)$ is a nondegenerate bilinear form on $\mathfrak{g}$, which is positive definite when restricted to $\mathfrak{a}$. Given $\Lambda$ in $\mathfrak{a}^{*}$, define $H_{\Lambda}$ to be the unique element in $\mathfrak{a}$ such that

$$
B\left(H_{\Lambda}, H\right)=\Lambda(H) \quad \forall H \in \mathfrak{a}
$$

and then an inner product $\langle\cdot, \cdot\rangle$ on $\mathfrak{a}^{*}$ by the rule

$$
\left\langle\Lambda, \Lambda^{\prime}\right\rangle=B\left(H_{\Lambda}, H_{\Lambda^{\prime}}\right) \quad \forall \Lambda, \Lambda^{\prime} \in \mathfrak{a}^{*} .
$$

The inner product on $\mathfrak{a}^{*}$ extends to a bilinear form, also denoted $\langle\cdot, \cdot\rangle$, on the complexification $\mathfrak{a}_{\mathbf{C}}^{*}$ of $\mathfrak{a}^{*}$. For every $\theta \in[0,1]$, we define the quadratic function $Q_{\theta}$ on $\mathfrak{a}_{\mathbf{C}}^{*}$ by the rule

$$
Q_{\theta}(\Lambda)=\langle\Lambda, \Lambda\rangle+(1-\theta)\langle\rho, \rho\rangle \quad \forall \Lambda \in \mathfrak{a}_{\mathbf{C}}^{*} .
$$

For any (complex-valued) linear form $\Lambda$ on $\mathfrak{a}$, let $\phi_{\Lambda}$ denote the correspondence elementary spherical function. Recall that $\phi_{\Lambda}$ is an eigenfunction for the (modified) Laplace-Beltrami operator and

$$
\mathscr{L}_{\theta} \phi_{\Lambda}=Q_{\theta}(\Lambda) \phi_{\Lambda} \quad \forall \Lambda \in \mathrm{a}_{\mathrm{C}}^{*} \quad \forall \theta \in[0,1] .
$$


Let $\mathbf{W}_{1}$ be the interior of the convex hull in $\mathfrak{a}^{*}$ of the images of $\rho$ under the Weyl group $W$ of $(\mathfrak{g}, \mathfrak{a})$. For $\delta$ in $(0,1)$, we denote by $\mathbf{W}_{\delta}$ and $\mathbf{T}_{\delta}$ the dilate of $\mathbf{W}_{\delta}$ by $\delta$ and the tube over the polygon $\mathbf{W}_{\delta}$, i.e., $\mathbf{T}_{\delta}=\mathfrak{a}^{*}+i \delta \mathbf{W}_{1}$.

If $\Lambda$ lies in $\mathbf{T}_{1}$, then $\phi_{\Lambda}$ is bounded. Moreover, let $p \in(1,2)$ and denote $\delta(p)=2 / p-1$; then if $\Lambda$ lies in $\mathbf{T}_{\delta(p)}$, then $\phi_{\Lambda}$ is in $L^{q}(M)$, for all $q \in$ $\left(p^{\prime}, \infty\right)$. The spherical Fourier transform $\tilde{f}$ of an $L^{1}(G)$-function $f$ is defined by the formula

$$
\tilde{f}(\Lambda)=\int_{G} f(x) \phi_{-\Lambda}(x) d x \quad \forall \Lambda \in \mathfrak{a}^{*} .
$$

The main result of this section is the following.

Theorem 2.2. Let $\theta \in(0,1)$ and $\alpha$ be admissible. Then the following hold:

(i) if $\operatorname{Re}(\alpha)<-1$, then $g_{\alpha, \theta}$ is bounded on $L^{p}(X)$ for all $p \in\left[p_{\theta}, p_{\theta}^{\prime}\right]$;

(ii) if $\operatorname{Re}(\alpha)>0$, then $g_{\alpha, \theta}$ is not bounded on $L^{p_{\theta}}(X)$ and on $L^{p_{\theta}^{\prime}}(X)$;

(iii) $g_{\alpha, \theta}$ is bounded on $L^{p}(X)$ for all $p \in\left(p_{\theta}, p_{\theta}^{\prime}\right)$.

Proof. The statements (i) and (iii) are an easy consequence of Corollary 1.2 and Theorem 1.5.

We now prove (ii). First, we show that $g_{\alpha, \theta}$ is not bounded on $L^{p_{\theta}^{\prime}}(X)$. Note that for every $\Lambda$ in the tube $\mathbf{T}_{\delta\left(p_{\theta}\right)}$, we have

$$
\begin{aligned}
\left\|g_{\alpha, \theta}\right\| \|_{p_{\theta}^{\prime}} & \geq\left\|g_{\alpha, \theta}\left(\frac{\phi_{\Lambda}}{\left\|\phi_{\Lambda}\right\|_{p_{\theta}^{\prime}}}\right)\right\|_{p_{\theta}^{\prime}} \\
& =(2 \pi)^{-1 / 2}\left(\int_{\mathbf{R}}|\Gamma(\alpha-i u)|^{2}\left|Q_{\theta}(\Lambda)^{-i u}\right|^{2} d u\right)^{1 / 2},
\end{aligned}
$$

which implies, say,

$$
\begin{aligned}
\left\|g_{\alpha, \theta}\right\| \|_{p_{\theta}^{\prime}} & \geq \sup _{\Lambda \in \mathbf{T}_{\delta\left(p_{\theta}\right)}}(2 \pi)^{-1 / 2}\left(\int_{\mathbf{R}}|\Gamma(\alpha-i u)|^{2}\left|Q_{\theta}(\Lambda)^{-i u}\right|^{2} d u\right)^{1 / 2} \\
& =\sup _{\Lambda \in \mathbf{T}_{\delta\left(\theta_{\theta}\right)}} A_{\alpha, \theta}(\Lambda) .
\end{aligned}
$$

Choose $\Lambda=\Lambda_{1}+i\left[\delta\left(p_{\theta}\right)-\left\langle\lambda_{1}, \Lambda_{1}\right\rangle\right] \rho$, where $\Lambda_{1} \in \mathfrak{a}^{*}$ (recall that $\delta\left(p_{\theta}\right)=$ $\left.2 / p_{\theta}-1\right)$. A simple calculation shows that

$$
Q_{\theta}(\Lambda)=\left|\Lambda_{1}\right|^{2}\left(1+2 \delta\left(p_{\theta}\right) b-\left|\Lambda_{1}\right|^{2} b\right)+2 i\left[\delta\left(p_{\theta}\right)-\left|\Lambda_{1}\right|^{2}\right]\left\langle\Lambda_{1}, \rho\right\rangle .
$$

Set $\Lambda_{1}=\lambda \rho$. Assume, for instance, that $\lambda \rightarrow 0+$. Recall that we have to estimate the integral $\int_{\mathbf{R}}|\Gamma(\alpha-i u)|^{2}\left|Q_{\theta}(\Lambda)^{i u}\right|^{2} d u$ from below. With our choice of the parameter $\Lambda$ the integrand is just

$$
|\Gamma(\alpha-i u)|^{2} \exp \left[-2 u \arctan \frac{2\left(\delta\left(p_{\theta}\right)-\lambda^{2} b\right)}{\lambda\left(1+2 \delta\left(p_{\theta}\right) b-\lambda^{2} b^{2}\right)}\right] .
$$

When $u \rightarrow-\infty$, it behaves like

$$
\exp (-\pi|u|)|u|^{2 \operatorname{Re}(\alpha)-1} \exp \left[-2 u\left(\pi / 2-\arctan \frac{\lambda\left(1+2 \delta\left(p_{\theta}\right) b-\lambda^{2} b^{2}\right)}{2\left(\delta\left(p_{\theta}\right)-\lambda^{2} b\right)}\right)\right],
$$
i.e.,

$$
|u|^{2 \operatorname{Re}(\alpha)-1} \exp \left[2 u \arctan \frac{\lambda\left(1+2 \delta\left(p_{\theta}\right) b-\lambda^{2} b^{2}\right)}{2\left(\delta\left(p_{\theta}\right)-\lambda^{2} b\right)}\right]=B_{\alpha, \theta}(u, \lambda)
$$


say. By the monotone convergence theorem we have that

$$
\lim _{\lambda \rightarrow 0+} \int_{\mathbf{R}} B_{\alpha, \theta}(u, \lambda) d u=+\infty ;
$$

thus

$$
\begin{aligned}
\left\|g_{\alpha, \theta}\right\| \|_{p_{\theta}^{\prime}} & =\sup _{\Lambda \in \mathbf{T}_{\delta\left(p_{\theta}\right)}} A_{\alpha, \theta}(\Lambda) \geq C \lim _{\lambda \rightarrow 0+} \int_{\mathbf{R}} B_{\alpha, \theta}(u, \lambda) d u \\
& =+\infty
\end{aligned}
$$

as required.

We now prove that $g_{\alpha, \theta}$ is not bounded on $L^{p_{\theta}}(X)$. For the rest of the proof we shall denote by $A$ the operator $\mathscr{L}_{\theta}$. For every function $\psi$ in the Schwartz space $\mathscr{S}(\mathbf{R})$ such that $\|\psi\|_{2} \leq 1$ we have that

$$
\begin{aligned}
\left\|g_{\alpha, \theta}(f)\right\|_{p_{\theta}} & \geq\left\|\int_{\mathbf{R}} \Gamma(\alpha-i u) A^{i u} f \psi(u) d u\right\|_{p_{\theta}} \\
& \geq\left|\int_{X} d x \frac{\phi_{\Lambda}(x)}{\left\|\phi_{\Lambda}\right\|_{p_{\theta}^{\prime}}} \int_{\mathbf{R}} \Gamma(\alpha-i u) A^{i u} f(x) \psi(u) d u\right|
\end{aligned}
$$

whenever $\Lambda$ is in the tube $\mathbf{T}_{\delta\left(p_{\theta}\right)}$. Now, we may interchange the order of integration. Indeed, since $A$ is bounded on $L^{p_{\theta}}(X)$,

$$
\begin{aligned}
& \int_{X} \int_{\mathbf{R}}\left|\frac{\phi_{\Lambda}}{\left\|\phi_{\Lambda}\right\|_{p_{\theta}^{\prime}}} \Gamma(\alpha-i u) A^{i u} f(x) \psi(u)\right| d u d x \\
& \quad \leq \int_{\mathbf{R}}|\Gamma(\alpha-i u) \psi(u)|\left\|A^{i u} f\right\|_{p_{\theta}} d u \\
& \quad \leq C\|f\|_{p_{\theta}} \int_{\mathbf{R}}|\Gamma(\alpha-i u) \psi(u)|\left\|A^{i u}\right\|_{p_{\theta}} d u \\
& \quad \leq C\|f\|_{p_{\theta}} \int_{\mathbf{R}}|\psi(u)|(1+|u|)^{\alpha} d u<\infty .
\end{aligned}
$$

Thus, by interchanging the order of integration, we obtain that

$$
\begin{aligned}
\left\|g_{\alpha, \theta}(f)\right\|_{p_{\theta}} & \geq\left|\int_{\mathbf{R}} \Gamma(\alpha-i u) \psi(u)\left\langle A^{i u} f, \frac{\phi_{\Lambda}}{\left\|\phi_{\Lambda}\right\|_{p_{\theta}^{\prime}}}\right\rangle d u\right| \\
& =\frac{|\tilde{f}(\Lambda)|}{\left\|\phi_{\Lambda}\right\|_{p_{\theta}^{\prime}}}\left|\int_{\mathbf{R}} \Gamma(\alpha-i u) Q_{\theta}(\Lambda)^{i u} \psi(u) d u\right| .
\end{aligned}
$$

We now take the supremum over all functions $\psi$ in the Schwartz space $\mathscr{S}(\mathbf{R})$ such that $\|\psi\|_{2} \leq 1$. We obtain that

$$
\left\|g_{\alpha, \theta}(f)\right\|_{p_{\theta}} \geq \frac{|\tilde{f}(\Lambda)|}{\left\|\phi_{\Lambda}\right\|_{p_{\theta}^{\prime}}}\left(\int_{\mathbf{R}}|\Gamma(\alpha-i u)|^{2}\left|Q_{\theta}(\Lambda)^{i u}\right|^{2} d u\right)^{1 / 2} .
$$

Recall that for every $\Lambda$ in the tube $\mathbf{T}_{\delta\left(p_{\theta}\right)}$ the continuous linear functional on $L^{p_{\theta}}(X)$ defined by the rule $f \mapsto \tilde{f}(\Lambda)$ is represented by the spherical function $\phi_{\Lambda}$, which is in $L^{p_{\theta}^{\prime}}(X)$. Therefore

$$
\sup _{\|f\|_{p_{\theta}=1}}|\tilde{f}(\Lambda)|=\left\|\phi_{\Lambda}\right\|_{p_{\theta}^{\prime}}
$$


Let $f_{\Lambda}$ denote the (unique) function in $L^{p_{\theta}}(X)$ which has norm one and "realizes" the norm of the functional, namely

$$
\left|\tilde{f}_{\Lambda}(\Lambda)\right|=\left\|\phi_{\Lambda}\right\|_{p_{\theta}^{\prime}} \cdot
$$

Then this particular function $f_{\Lambda}$ satisfies the following inequality

$$
\left\|g_{\alpha, \theta}\left(f_{\Lambda}\right)\right\|_{p_{\theta}} \geq\left(\int_{\mathbf{R}}|\Gamma(\alpha-i u)|^{2}\left|Q_{\theta}(\Lambda)^{i u}\right|^{2} d u\right)^{1 / 2}
$$

so that

$$
\begin{aligned}
\left\|\mid g_{\alpha, \theta}\right\| \|_{p_{\theta}} & =\sup _{\|f\|_{p_{\theta}=1}}\left\|g_{\alpha, \theta}(f)\right\|_{p_{\theta}} \geq \sup _{\Lambda \in \mathbf{T}_{\delta\left(\theta_{\theta}\right)}}\left\|g_{\alpha, \theta}\left(f_{\Lambda}\right)\right\|_{p_{\theta}} \\
& \geq \sup _{\Lambda \in \mathbf{T}_{\delta\left(p_{\theta}\right)}}\left(\int_{\mathbf{R}}|\Gamma(\alpha-i u)|^{2}\left|Q_{\theta}(\Lambda)^{i u}\right|^{2} d u\right)^{1 / 2} .
\end{aligned}
$$

We have already proved that this supremum equals $+\infty$, thereby concluding the proof of (ii).

The proof of the theorem is now complete.

\section{REFERENCES}

1. J. Ph. Anker, Sharp estimates for some functions of the Laplacian on noncompact symmetric spaces, Duke Math. J. 65 (1992), 257-297.

2. R. R. Coifman, R. Rochberg, and G. Weiss, Applications of transference: The $L^{p}$ version of von Neumann's inequality and Littlewood-Paley-Stein theory, Linear Spaces and Approximation (P. L. Butzer and B. Sz.-Nagy, eds.), Birkhäuser-Verlag, Basel, 1978, pp. 53-67.

3. M. G. Cowling, Harmonic analysis on semigroups, Ann. of Math. 117 (1983), 267-283.

4. __ Square functions in Banach spaces, Miniconference in Linear Analysis and Function Spaces, Proc. Centre for Math. Anal. (A. McIntosh and A. Pryde, eds.), vol. 9, Australian National University, 1985.

5. M. G. Cowling, I. Doust, A. McIntosh, and A. Yagi, Banach space operators with a bounded $H_{\infty}$ functional calculus, J. Austral. Math. Soc. (to appear).

6. M. G. Cowling, S. Giulini, and S. Meda, $L^{p}-L^{q}$ estimates for functions of the LaplaceBeltrami operator on noncompact symmetric spaces. I, Duke Math. J. (to appear).

7.,$- L^{p}-L^{q}$ estimates for functions of the Laplace-Beltrami operator on noncompact symmetric spaces. II, preprint.

8. S. Helgason, Groups and geometric analysis, Academic Press, New York, 1984.

9. N. Lohouè, Estimation des fonctions de Littlewood-Paley-Stein sur les variétés Riemanniennes à courbure non positive, Ann. Sci. Ėcole Norm. Sup. Paris 20 (1987), 505-544.

10. E. M. Stein, Topics in harmonic analysis related to the Littlewood-Paley theory, Ann. of Math. Studies, no. 63, Princeton Univ. Press, Princeton, NJ, 1970.

11. $\ldots$ The development of square functions in the work of A. Zygmund, Bull. Amer. Math. Soc. 7 (1982), 359-376.

ITALIA

Dipartimento di Matematica, Politecnico di Milano, via Bonardi 9, 20133 Milano,

E-mail address: stemed@ipmma1.polimi.it 